\title{
Microarqueología Aplicada al Análisis e Interpretación de Termoalteraciones en Restos Óseos de Tortugas de Sitios Arqueológicos en el Caribe Colombiano
}

\author{
Microarchaeology Applied to the Analysis and Interpretation of Thermal Alterations in \\ Faunal Remains from Archaeological Sites in the Colombian Caribbean \\ Elizabeth Ramos Roca ${ }^{i}$ Lina María Campos Quintero ${ }^{i}$
}

\begin{abstract}
RESUMEN
En este artículo se discuten los resultados de un primer estudio piloto dirigido al análisis de restos óseos termoalterados como indicador de prácticas culturales en el contexto de la arqueología de la Región Caribe de Colombia. Este tipo de modificaciones, son comunes en las muestras arqueológicas de la región, y su investigación ofrece un gran potencial para indagar en el tema de las prácticas alimentarias entre otros aspectos. Para el estudio se utilizaron muestras de tortuga hicotea (Trachemys callirostris callirostris) recuperadas en excavaciones arqueológicas realizadas recientemente en el sitio de Cacaramoa, municipio de Sabanagrande (Atlántico, Colombia), y fragmentos óseos modernos de tortuga boba (Caretta caretta), analizadas con la técnica del Espectrofotómetro Infrarrojo por Transformada de Fourier (FTRI). En esta investigación se corroboraron los problemas que subyacen a la aplicación de las mismas categorías para termoalteración sin considerar las particularidades de la estructura anatómica de las distintas especies de tortugas, además de factores como el tiempo de exposición al fuego y las variaciones que en relación con esta variable pueden observarse entre las distintas especies. Como resultado fue posible generar un primer referente propio para la investigación de termoalteraciones en restos de tortuga en esta región.
\end{abstract}

Palabras clave: Zooarqueología, Tafonomía, Quelonios, Termoalteraciones, FTIR.

\section{ABSTRACT}

This article discusses the results of the first pilot study directed to the analysis of thermoaltered bones as an indicator of cultural practices in the context of Caribbean archaeology. These types of modifications are common in the archaeological samples of the region and its research offers great potential to investigate dietary practices among other things. In this sense, samples from slider turtle (Trachemys callirostris callirostris) recently recovered from

\footnotetext{
i Departamento de Antropología, Universidad de los Andes, Bogotá Colombia. Correo-e: eramosroca@uniandes.edu.co; lina16112005@ gmail.com.

Recibido: 08-05-2013 - Revisado: 03-01-2014 - Aceptado: 21-03-2014
} 
archaeological excavations at the site of Cacaramoa, municipality of Sabanagrande (Atlántico, Colombia), and bone fragments from modern Boba turtle (Caretta caretta), were analyzed through Fourier Transform Infrared Spectroscopy (FTIR) technique. This research confirmed the issues underlaying the application in different cases of the same categories for thermal modifications without taking into account the peculiarities of each geographical context and the differential changes that may occur in diverse species.As a result, it was possible to generate a first local referent for the study of thermal modifications in turtle remains for this region.

Key words: Microarchaeology, Zooarchaeology, Taphonomy, Thermal Alterations, FTIR

\section{INTRODUCCIÓN}

Dentro de las modificaciones que se observan en los restos óseos arqueológicos como producto de los diversos eventos ligados a las prácticas alimentarias, una de las que se presentan con mayor frecuencia son las modificaciones por fuego o termoalteraciones (hervido, asado, cocido, etc.), las cuales constituyen una importante línea de evidencia. Adicionalmente, las termoalteraciones pueden también ser el resultado de otros eventos como las prácticas de descarte y lo su uso como combustible (Gifford Gonzáles 1989, Lyman 1994).

No obstante, uno de los problemas arqueológicos fundamentales ligados a la reconstrucción de las actividades de subsistencia en general,y de las prácticas alimentarias en particular, ha sido la dificultad para identificar el tipo específico de actividades que las modificaciones por fuego reflejan y que usualmente malinterpretamos o no logramos observar debido a la ausencia de metodologías y técnicas adecuadas. Un factor de sesgo importante para considerar en este sentido, es el hecho de que aplicamos las mismas categorías para el análisis de termoalteraciones a las muestras arqueológicas provenientes de distintos sitios y en los huesos de distintas especies, desconociendo que aun dentro de una misma área geográfica estas pueden variar como consecuencia de las condiciones particulares como son el tipo y tiempo de exposición al fuego, el combustible utilizado, $y$ principalmente las diferencias morfológicas y de composición ósea en distintos taxones y elementos anatómicos. Es precisamente este problema el que nos llevó a proponer un primer estudio para contribuir a documentar la naturaleza de los cambios ocurridos en los huesos de animales por el uso del fuego en las distintas actividades humanas ligadas a la alimentación en un contexto geográfico determinado, en este caso la Región Caribe de Colombia.

\section{ANTECEDENTES}

Entender la relación entre los distintos tipos de termoalteración ósea y las prácticas relacionadas con la alimentación, ha sido un tema recurrente de investigación en países como Estados Unidos y en el Reino Unido desde la década de los ochentas. Estas investigaciones han estado encaminadas a caracterizar, con base en la quema experimental de huesos modernos y el estudio de la morfología del hueso a nivel microscópico, los distintos tipos de termoalteración producidos por diferentes tratamientos o exposiciones del hueso. Los estudios de Shipman et al. (1984), Buikstra y Swegle (1989) y Munro et al. (2007), que identifican rangos generales de clasificación con criterios macroscópicos tales como coloración, fragmentación, erosión de la superficie, entre otros, se han convertido en importantes referentes en este tema. Tras la revisión de estos trabajos y la realización de un estudio previo sobre termoalteración en restos óseos en Colombia (Campos 20I2), se han identificado tres problemas metodológicos en lo que concierne a la caracterización del material óseo termoalterado.

El primero está relacionado con el hecho de que la coloración, la fragmentación, la erosión de la superficie, etc. de los huesos, no son indicadores exclusivos de modificaciones térmicas. Procesos tafonómicos tales como la intemperización y los procesos diagenéticos (fosilización y tinciones por sedimentos), pueden evidenciar estas mismas características y por tanto, no son criterios confiables de análisis en este campo (Nicholson 1993,Von End y Ortner y Ortner 1984, Shahack et al. 1997).

El segundo, es que "no siempre dos huesos con la misma exposición de calor y la misma duración, tienen una misma coloración" (Nicholson 1993: 425-427). En este sentido, no es posible hacer 
asociaciones basadas exclusivamente en el color, debido a que otras condiciones como estado de preservación, antigüedad o especie a la que pertenece el hueso (entre otros), pueden interferir en las interpretaciones.

Por último, los criterios macroscópicos que surgen de los estudios mencionados se han tomado como referentes para la clasificación de restos óseos termoalterados provenientes de contextos geográficos diferentes. Así, estos criterios fueron elaborados con base en muestras arqueológicas específicas y su aplicación en otros contextos con características distintas, puede generar importantes sesgos en la interpretación.

En consecuencia con lo anterior, el potencial que esta evidencia ofrece para la interpretación de los patrones alimentarios del pasado se ve significativamente reducido debido a que no se puede discernir con claridad las causas de las termolteraciones y los posibles eventos asociados con las mismas. Teniendo en cuenta las inconsistencias arriba señaladas resulta evidente la importancia de realizar estudios con muestras de variados contextos arqueológicos y con las distintas especies. Es necesario además, que a nivel microscópico se analicen elementos distintos (colágeno tipo I, cristales de hidroxiapatita, eliminación progresiva del agua, etc.), en diferentes escalas de microscopía del hueso y con técnicas especializadas.

Globalmente hay un amplio conjunto de trabajos sobre microscopía aplicada al análisis de las termoalteraciones (Nicholson 1993, Koon et al. 2003, Koon et al. 2010; Lebon et al. 2008, Weiner 2010, Asscher y Boaretto 20II, Nevin et. al. 20I2). En el escenario latinoamericano, son escasos los estudios sobre alteración térmica de restos óseos de fauna, predominando los trabajos donde se realiza la caracterización de estas a nivel macroscópico y por lo general en el marco de investigaciones sobre subsistencia y prácticas de consumo (Ávido 2010, Ariel y Paunero 2008, Simonetti y Cornejo 199I). En algunas de estas investigaciones, sin embargo, se han implementado también técnicas especializadas a nivel microscópico, particularmente desde la arqueología y desde la física aplicada que hacen uso de técnicas como: XRD, SAXS, SEM, TGA y DSC (Pijoan et al. 2007, Lozano et al. 2003 y Campos 2012).

En este contexto, este artículo busca aportar a la discusión sobre la aplicabilidad de algunas técnicas especializadas para el análisis del registro microscópico, particularmente en relación con el estudio de termoalteraciones en restos óseos de tortugas arqueológicas y modernas'.

\section{USO DEL FTIR PARA EL ANÁLISIS DE TERMOALTERACIONES ÓSEAS}

Con el objetivo de monitorear, a nivel microscópico, los cambios de la fase mineral causados por la termoalteración, se hizo uso de la Espectroscopía Infrarroja de Transformada Fourier (FTIR). Esta técnica permite el análisis de la composición química de los materiales y la caracterización de su orden atómico; consiste en la emisión de un rayo de luz infrarrojo que atraviesa las muestras y que genera un espectro de absorbancia. Cuando un hueso está termoalterado, la matriz orgánica se oxida y la fase mineral se recristaliza (Weiner 2010: II7). Esto repercute en un cambio en el orden atómico y tamaño de los cristales, al igual que en la destrucción de la matriz orgánica ${ }^{2}$. Debido a que el efecto de la diagénesis ${ }^{3}$ en los huesos también produce cambios similares a nivel microscópico, el FTIR permite la caracterización diferencial de los cambios en la matriz mineral producidos por ambas condiciones (termoalteración y modificaciones diagenéticas). Tres referentes de los espectros infrarrojos se tuvieron en cuenta para el análisis de la cristalinidad de los huesos como indicador de termoalteración.

El primer referente, está relacionado con el fin principal de la espectroscopia infrarroja, la caracterización de distintos elementos a partir de mirar en qué longitudes de onda los enlaces de las moléculas están absorbiendo los rayos infrarrojos. En la Figura I, tomada de Weiner (2010: 195), el autor ilustra el cambio de los espectros específicamente de los picos de la matriz orgánica, los picos de carbonato y los picos de fosfato de acuerdo a un código entre 0 y 6 , donde 6 , representa una mayor termoalteración. 


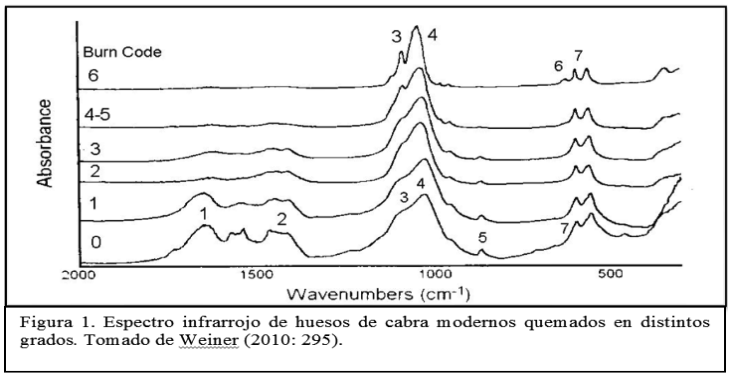

Figura 1: Espectro infrarrojo de huesos de cabra modernos termoalterados a distintos grados de temperatura en un rango de 0 a 6 , donde 0 es no termoalterado y 6 muy termoalterado. Tomado de Weiner $(2010,295)$.

Figure 1: Infrared spectra of modern goat bones burned at different temperature degrees in a range from 0 to 6 where 0 is not burned and 6 is very burned. Taken from (Weiner 2010, 295).

El segundo referente, está relacionado con determinar la cristalinidad de un hueso a partir del factor de división o Splitting Factor (SF) ${ }^{4}$. El factor de división (SF) permite caracterizar la cristalinidad del carbonato de hidroxiopatito biogénico (Asscher y Boaretto 20II) a partir de diferenciar el efecto del orden. Este valor (SF) mide el carbonato como factor de desorden, es decir que en un hueso termoalterado a medida que el SF aumenta, los cristales son más grandes y el desorden de la matriz cristalina se potencializa.

Finalmente, el tercer referente es el ancho a media altura o Full Width at Half Height (FWHH) del pico de fosfato ubicado a $1035 \mathrm{~cm}^{-1}\left(\mathrm{~V}_{3} \mathrm{PO}_{4}\right)$, que refleja el grado en que la red del enlace químico es homogénea. Asscher y Boaretto (20II) analizaron como el ancho a media altura de dicho pico refleja tanto el desorden atómico como el tamaño de las partículas de la muestra ${ }^{5}$. Este indicador permitió ver el efecto del desorden atómico causado por la termoalteración y entender la incidencia de la molienda de la muestra en le emisión de espectros infrarrojos.

\section{APLICACIÓN DEL FTIR A LA INVESTIGACIÓN ARQUEOLÓGICA EN EL CARIBE COLOMBIANO: METODOLOGÍA}

Teniendo en cuenta estos tres referentes para el análisis de termoalteraciones óseas, se aplicó la técnica de Espectroscopía Infrarroja de Transformada Fourier en dos eventos principalmente: uno de experimentación con fragmentos óseos modernos de caparazón de tortuga Caretta Caretta y otro de análisis de fragmentos óseos termoalterados de caparazón de tortuga Trachremys callirostris callirostris (Región Caribe de Colombia), recuperados en el Corte I del sitio de Cacaramoa.

El objetivo principal del ejercicio de experimentación fue por un lado evaluar la aplicabilidad de la técnica FTIR y por otro, evidenciar los cambios de comportamiento de los espectros y los valores de SF y FWHH para altas y bajas temperaturas en restos óseos modernos de tortuga Caretta caretta. Como resultado se pudo generar un referente de comparación para la muestra arqueológica de tortuga Trachremys callirostris callirostris (hicotea).

Un grupo de fragmentos óseos se sometieron a temperaturas controladas de $200^{\circ} \mathrm{C}$, $400^{\circ} \mathrm{C}, 500^{\circ} \mathrm{C}$ y $600^{\circ} \mathrm{C}$ durante 4 horas, mientras que otros, se hirvieron en agua durante el mismo tiempo. Posteriormente, se realizó la extracción de espectros infrarrojos por rangos de temperaturas y se elaboró la Curva de Molido o Grinding curve.

Por medio de la emisión de espectros repetidos para cada rango de temperatura, se logró caracterizar patrones de comportamiento de los picos. La Figura 2 muestra el ejemplo de un fragmento termoalterado a $600^{\circ} \mathrm{C}$ con la descripción de los cambios de dichos picos, generados por la modificación térmica de los huesos.

Para realizar la Curva de Molido o Grinding Curve, para cada fragmento se tomó una micro-parte de material óseo y se molió repetidas veces en un mortero de ágata (con $\mathrm{KBr}$ ); por cada molienda del mismo material, se sacó un espectro infrarrojo. Cuando se graficaron los valores SF y $\mathrm{FWHH}$, fue posible generar líneas de tendencia que reflejaron el efecto del tamaño de la partícula.Adicionalmente, con la ubicación en la gráfica del FWHH y el SF, fue posible evidenciar los efectos de desorden atómico (Asscher et al. 20II). 


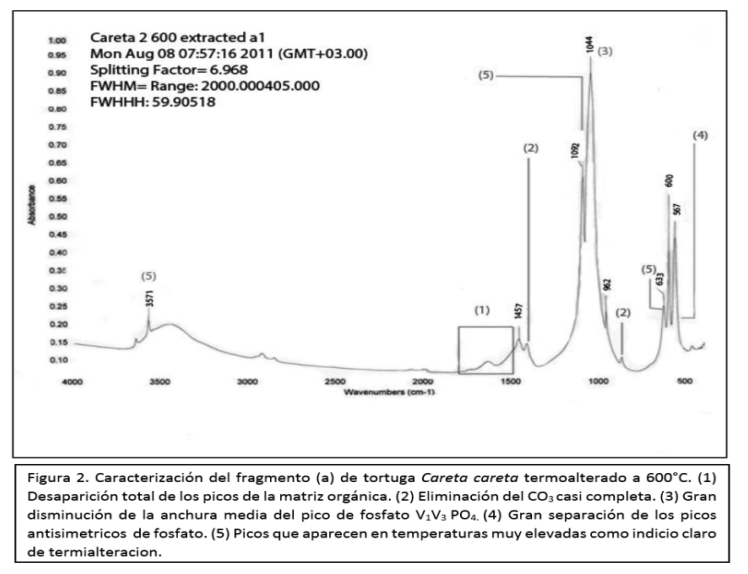

Figura 2: Caracterización de fragmento de tortuga Careta careta termoalterado a $600^{\circ} \mathrm{C}$. (1) Desaparición total de los picos de la matriz orgánica, (2) Eliminación del $\mathrm{CO}_{3}$ casi completa, (3) Gran disminución de la altura media del pico de fosfato $\mathrm{V}_{1} \mathrm{~V}_{3} \mathrm{PO}_{4},(4)$ Gran separación de los picos antisimetricos de fosfato y (5) Picos que aparecen a temperaturas elevadas como indicio claro de termoalteración.

Figure 2: Characterization of turtle Careta careta bone fragment burned at $600^{\circ} \mathrm{C}$. (1) Complete vanishing of organic matrix peaks, (2) Almost complete $\mathrm{CO}_{3}$ removal, (3) Decreasing of phosphate peak $V_{1} V_{3} P_{4}$ at half-height, (4) Splitting of antisymmetric phosphate peaks and (5) Peaks characteristic of high temperatures of burning, indicative of thermal alteration.

Se observó que a medida que aumenta la temperatura de quema para los fragmentos de caparazón modernos (a partir de $400^{\circ} \mathrm{C}$ ) hay un desplazamiento de las líneas de tendencia indicando cristales más ordenados a nivel atómico-disminución en el eje $(x)$ y aumento del eje $(y)$ - debido a las substituciones de carbonato, al crecimiento del tamaño de los cristalesii dado por el aumento en los valores de SF y a la relativa disminución del tamaño de las partículas asociada con la disminución del ancho a media altura del pico de fosfato $\mathrm{V}_{1} \mathrm{~V}_{3} \mathrm{PO}_{4}$.

En el caso de la muestra arqueológica de tortuga hicotea (Trachemys scripta callirostris) del sitio de Cacaramoa, para la preparación del material y extracción de espectros, se utilizó el mismo procedimiento que se tuvo con la muestra experimental moderna. Estos espectros, evidenciaron una diferencia entre aquellos fragmentos que por coloración parecían termoalterados pero no lo eran y los que en efecto, estaban termoalterados. En la Figura 3, se ilustra la diferencia entre las características de los picos de un hueso termoalterado (como el gris) y las características de los picos de un hueso no termoalterado (como el marrón).

De igual manera, estos fragmentos se graficaron en la Curva de Molido. Esta curva de molido evidenció la distribución de los fragmentos por coloraciones, el carbonato como factor de desorden y el tamaño de los cristales a partir del $\mathrm{SF}$ y el FWHH. Estos indicadores son típicos de la termoalteración en restos óseos.

\section{RESULTADOS}

A partir del ejercicio de experimentación y el análisis de huesos arqueológicos fue notoria la dificultad del uso de la técnica de FTIR para análisis de huesos termoalterados a bajas temperaturas (huesos hervidos y quemas inferiores a $400^{\circ} \mathrm{C}$ ). Particularmente, se hizo evidente el gran aporte del FTRI para ver cambios en la matriz mineral (a partir de $450^{\circ} \mathrm{C}$ ) pero no para observar la temprana descomposición del tercio orgánico. A pesar de estas limitaciones, se lograron distinguir claramente cuatro eventos: I) no todos los restos óseos de coloración negra estaban termoalterados; su coloración es producto de tinciones por óxidos del suelo; 2) los fragmentos óseos de coloración marrón, por el contrario a lo que pensábamos antes de correr el análisis, tampoco están temoalterados, su coloración es efecto de la acidez del suelo; 3 ) fue posible distinguir rangos de temperatura que son transición al estado de calcinación y que en el caso del estudio toman coloraciones entre grises y azules y 4) existen casos mixtos donde el análisis de un mismo hueso en partes corticales opuestas, sugiere que en una de sus superficies fue termoalterado y en la otra teñido por efectos del suelo. 


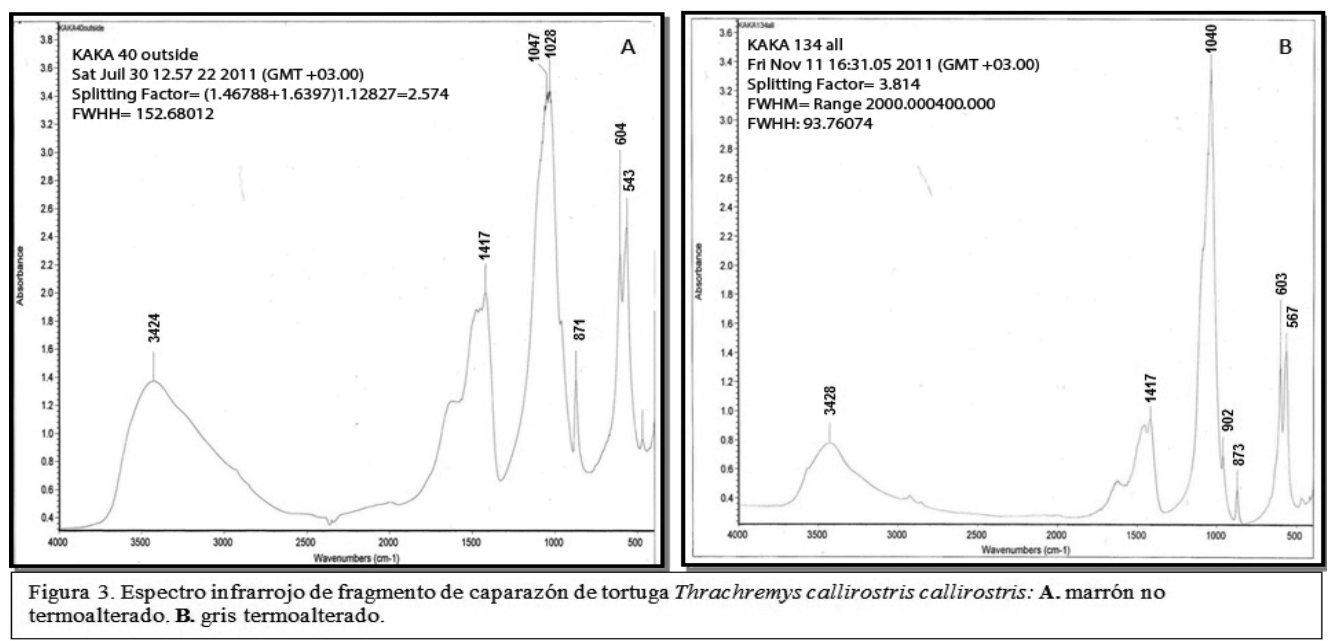

Figura 3: Espectro infrarrojo de fragmentos de caparazón de tortuga Thrachremys callirostris callirostris. (A) fragmento de hueso marrón, no termoalterado. (B) fragmento de hueso gris, termoalterado.

Figure 3: Infrared spectrum of Thrachremys callirostris callirostris turtle shell fragments. (A) brown bone fragment, not burned. (B) grey bone fragment, burned.

\section{CONCLUSIONES $Y$ PERSPECTIVAS DE INVESTIGACIÓN}

Uno de los principales retos que enfrenta la investigación arqueológica en Colombia como en otras partes del continente y el mundo, es el de generar referentes propios para el análisis e interpretación de contextos arqueológicos particulares. Para el caso específico de la zooarqueología, las investigaciones de carácter tafonómico y diagenético resultan de particular importancia, ya que las conclusiones e interpretaciones derivadas de este tipo de análisis se ven por lo general fuertemente afectadas por estos procesos.

En este contexto, los resultados del trabajo que aquí se discutieron permitieron concluir en primer lugar, que las categorías de termoalteración a nivel macroscópico y microscópico propuestas para otras regiones, no necesariamente pueden ser aplicadas a los sitios que estamos investigando y que por lo tanto, es necesario continuar desarrollando técnicas de análisis apropiadas y ajustadas a las particularidades de cada región.

La experimentación realizada y las técnicas aplicadas en este estudio brindaron herramientas importantes para identificar en los huesos de las tortugas seleccionadas, por una parte, los diferentes tipos de cambios generados por el proceso de exposición al fuego y por otra, caracterizar cuáles de estos cambios pueden haber sido producidos por otros factores tafonómicos y diagenéticos y ser confundidos con eventos de modificación térmica. De particular relevancia resultó el evidenciar que los procesos de exposición al fuego a relativamente bajas temperaturas (entre 90 y 400 grados) como es el caso de los cocidos o hervidos -tan importantes para el estudio de las prácticas alimentarias en el pasado- no son fácilmente identificables en las muestras tanto a nivel macroscópico como microscópico.

Un resultado importante lo constituye la identificación de aquellos campos específicos en los que debemos seguir trabajando para implementar de forma más adecuada la técnica de FTIR según los requerimientos de cada contexto de investigación y contemplar otras posibles alternativas para medir las modificaciones que requieren del uso de otras técnicas complementarias para su análisis.

En este sentido, y como consecuencia de los resultados de este primer estudio piloto, recientemente, además de continuar con el FTIR, estamos incorporando el uso de otras técnicas especializadas a nivel microscópico como el SEM, TEM, TGA, con el objetivo de aproximarnos con mayor precisión los cambios a bajas temperaturas que están afectando directamente el tercio orgánico 
de los huesos de tortugas y la incidencia de las modificaciones tafonómicas en el estudio de las termoalteraciones.

Finalmente, es importante resaltar que si bien este estudio se concentró en especies particulares de tortugas, metodológicamente es aplicable para otras clases y especies animales, de allí su relevancia en el contexto de las discusiones zooarqueológicas en general.

\section{AGRADECIMIENTOS:}

Las autoras queremos expresar nuestra gratitud al Departamento de Antropología de la Universidad de los Andes por el apoyo logístico a esta investigación. Al Dr. Steven Weiner del Weizmann Institute of Science en Israel por la asesoría académica y el apoyo logístico brindado durante la fase experimental en el laboratorio. A la Fundación de investigaciones arqueológicas Nacionales (FIAN) por proporcionar una parte de los recursos para la realización del proyecto. Al equipo de colegas en la Universidad de Chile, quienes organizaron el II Encuentro Latinoamericano de Zooarqueología, marco dentro del cual se presentó este trabajo y quienes posteriormente se dieron a la tarea de hacer posible esta publicación.

\section{Notas}

I Este trabajo se realizó en el marco del programa de investigación "Arqueología, Medioambiente y Adaptación Humana en el Caribe colombiano" (Ramos 2006), que busca contribuir al entendimiento de las estrategias adaptativas de las poblaciones del Caribe colombiano, mediante la reconstrucción de los cambios en los patrones alimentarios a través del tiempo.

2 Cabe notar que los efectos de la termoalteración en las propiedades orgánicas y cristalinas del hueso ocurren a distintas temperaturas de acuerdo a las condiciones específicas de este (estado de preservación, antigüedad, especie a la que pertenece el hueso, etc.).

3 Se refiere al ambiente acumulativo químico y biológico que modifican el producto orgánico y las características estructurales del tercio orgánico del material óseo.

4 El valor surge del grado de división de los picos ubicados en $605 \mathrm{~cm}^{-1}$ y $567 \mathrm{~cm}^{-1}$

5 Es decir, que a mayor desorden atómico el pico será más ancho, y simultáneamente, más estrecho, en la medida que se disminuye el tamaño de las partículas.

\section{BIBLIOGRAFÍA}

Ariel, F y R. Paunero. 2008. "Análisis de la alteración térmica de los restos óseos procedentes del componente temprano de Cerro Tres Tetas (Meseta Central de Santa Cruz). Evidencia arqueológica y estudios experimentales". Arqueología de la Patagonia, una mirada desde el último confin, pp: I-I 3.

Asscher, Y. y, S. Boaretto. 201 I. "Variations in Atomic Disorder in Biogenic Carbonate Hydroxyapatite Using Infrared Spectrum Grinding Curve Method". Advanced Functional Materials 21:3308-3313.

Ávido, D. 2010. Una aproximación antropológica a alimentación de cazadores recolectores del Holoceno tardío en la Pampa Deprimida: el caso del sitio 'El Divisadero monte 6' (Partido de General Lavalle). Proyecto de Tesis de Licenciatura. Universidad de Buenos Aires. Manuscrito en posesión del autor.

Buikstra, J. E. and M. Swegle. 1989. "Bone modification due to burning: Experimental evidence". En Bone Modification, editado por R. Bonnichseny M.H. Sorg, M.Hpp. 247258. University of Maine, Orono.

Campos, L. 2012. Uso del Espectrofotometro infrarrojo con transformada de furias para el estudio de termoalteración de restos óseos de tortuga Hicotea. Monografía para optar al grado de Antropóloga, Departamento de Antropología, Universidad de los Andes, Bogotá.

Gifford-Gonzales, D.P. 1989. "Ethnographic analogues for interpreting modified bones: some cases from East Africa". En Bone Modification, editado por R. BonnichsenyM.H. Sorg, Pp. 179246. University of Maine, Orono.

Koon, H.E.C., R.A. Nicholson y M.J. Collins. 2003. "A practical approach to the identification of low temperature heated bone using TEM". Journal of Archaeological Science 30(I I): I393-1399.

Koon H.E.C., Collins M., O'Connor, T. and Covington T. 2010. "Sorting the butchered from the boiled". Journal of Archeaeological Science 37 (I): 62-69.

Lebon, M. Reiche, I. Fröhlich, F. Bahain, J. Falguéres, C. 2008. "Characterization of archaeological burnt bones: contribution of a new analytical protocol based on derivative FTIR spectroscopy and curve fitting of the VIV3 PO4 domain". Anal Bioanal Chem 392: I479-I 488.

Lozano, L. Peña, M., Heredia, A. Ocotlán y J. Gómez A. 2003.“"Thermal analysis study of human bone”.Journal of Materials Science 38: 4777-4782.

Lyman, R. Lee. 1994. Vertebrate Taphonomy. Cambridge University Press, New York.

Munro, L. Longstaffe y F. White C. 2007. "Burning and boiling of modern deer bone: Effects on cristallinity and oxygen isotope composition of biopatite phosphate". Paleogeography, Paleoclimatology, Paleoecology 249: 90-102.

Nevin, A., G Spoto y D. Anglos, D. 2012. "Laser spectroscopies for elemental and molecular analysis in art and archaeology. Applied Physics A - Materials”. Science \& Processing 106: 339-36I.

Nicholson, R.A. 1993. "A morphological Investigation of Burnt Animal Bone and an Evaluation of its Utility in Archaeology". Journal of Archaeological Science 20(4): 4I I-428.

Pijoan, C. J. Mansilla, I. Leboreiro, V. Lara y P. Bosch. 2007. "Thermal alterations in archaeological bones". Archeometry 49(4): 713-727.

Ramos, E. 2006. Arqueología, prácticas alimentarias y biodiversidad en el Caribe colombiano". Programa de investigación. Manuscrito en posesión del autor. 
Shahack, R., Y. Ofer Bary y S. Weiner. 1997. "Black coloured bones in Hayonim Cave, Israel: Differentiating Between Burning and Oxide staining". Journal of archaeological Science 24: 493-446.

Simoneti, J. y L. Cornejo. 1991.“Archaelogical Evidence of Rodent Consumption in Central Chile". Latin American Antiquity 2: 92-96.
Shipman, P., G. Foster y M. Schoeninger. 1984. "Burnt Bones and Teeth: An Experimental Study of Color, Morphology, Crystal".Journal of archaeological science I4(4): 307-325

Von Endt, D. y J. Ortner. 1984. "Experimental Effects of Bone Size and Temperature on Bone Diagenesis". Journal of Archaeological Science 8(3): 247-253.

Weiner, S. 2010. Microarchaeology: Beyond the Visible Archaeological Record. Cambridge University Press, New York. 